\title{
STABILITY OF TWO-DIMENSIONAL PARALLEL FLOWS FOR THREE-DIMENSIONAL DISTURBANCES*
}

\author{
BY CHIA-SHUN YIH (State University of Iowa)
}

The object of this note is to establish a relationship between the stability of twodimensional parallel flows for three-dimensional disturbances and that for two-dimensional ones. The special case of confined flow of a homogeneous fluid has been considered by Squire ${ }^{1}$. In the present note neither is the upper surface of the fluid necessarily assumed to be fixed, nor are the gravitational force and variations in density and viscosity neglected. The variations in density and viscosity, which for two-dimensional flow can occur only in the direction normal to the plane boundary along which the fluid flows, may be continuous or discontinuous.

For the disturbance, a stream function of the type

$$
\psi^{\prime}=\varphi(y) \exp i(m x+n z-m c t)
$$

can be taken, in which $x$ is measured in the direction of the primary flow, $y$ is measured in the direction normal to the plane boundary, $z$ is taken along an axis normal to the $x, y$ plane, and $m, n$, and $c$ are constants. If a rotation about the $y$-axis is performed so that the $x^{\prime}$-axis has the direction numbers $(m, 0, n)$ with respect to the original coordinate system, then

$$
m x+n z=m^{\prime} x^{\prime}
$$

in which $m^{\prime}=\left(m^{2}+n^{2}\right)^{1 / 2}$. If, furthermore, $c^{\prime}$ is defined by $m^{\prime} c^{\prime}=m c$, Eq. (1) can be written as

$$
\psi^{\prime}=\varphi(y) \exp i m^{\prime}\left(x^{\prime}-c^{\prime} t\right)
$$

which represents a two-dimensional disturbance progressing in the $x^{\prime}$-direction with wave number $m^{\prime}$ and celerity $c^{\prime}$. Similarly, the stream function

$$
\psi^{\prime \prime}=\varphi(y) \exp i(m x-n z-m c t)=\varphi(y) \exp i m^{\prime}\left(x^{\prime \prime}-c^{\prime} t\right)
$$

represents the same disturbance progressing in the $x^{\prime \prime}$-direction with direction numbers $(m, 0,-n)$.

Now the stream function

$$
\psi=\psi^{\prime}+\psi^{\prime \prime}=2 \varphi(y) \cos (n z) \exp i m(x-c t)
$$

represents a three-dimensional disturbance progressing in the $x$-direction with celerity $c$ and having wave numbers $m$ and $n$ in the $x$ - and $z$-directions, respectively. Since by symmetry $\psi^{\prime}$ and $\psi^{\prime \prime}$ are physically identical, it follows that the flow is stable or unstable for $\psi$ according as it is stable or unstable for $\psi^{\prime}$, because the differential system governing stability is linear and homogeneous in the quantity representing the stream function of the disturbance, so that the rule of superposition applies.

Now if the Reynolds number, slope, and pressure gradient of the primary flow are

*Received February 10, 1954.

${ }^{1} \mathrm{H}$. B. Squire, On the stability for three-dimensional disturbances of viscous fluid flow between parallel walls, Proc. Roy. Soc. London A142, 621-628 (1933). 
denoted respectively by $R, s$, and $\partial p / \partial x$, those for that component of the primary flow pertinent to $\psi^{\prime}$, denoted by $R^{\prime}, s^{\prime}$, and $\partial p^{\prime} / \partial x^{\prime}$, are determined by

$$
m^{\prime} R^{\prime}=m R, \quad m^{\prime} s^{\prime}=m s, \quad \frac{\partial p^{\prime}}{\partial x^{\prime}} \csc \beta^{\prime}=\frac{\partial p}{\partial x} \csc \beta
$$

in which $\beta$ and $\beta^{\prime}$ are the angles of inclination of the boundary to the horizontal in the $x$ - and $x^{\prime}$-directions, respectively, so that $s=\tan \beta$ and $s^{\prime}=\tan \beta^{\prime}$. As can be easily seen, the cross flow (in the $z^{\prime}$-direction) for the case of $\psi^{\prime}$ makes no consequential contribution to either the equations of motion or the equation of continuity, and does not affect in any way the satisfaction of the boundary conditions. Hence, the primary flow is stable or unstable for a three-dimensional disturbance according as it is stable or unstable for a two-dimensional one at a lower Reynolds number, a milder slope, and a reduced pressure gradient: the laws of reduction for the three quantities being given by Eqs. (6).

The writer arrived at the foregoing conclusion by using Squire's approach. Whereas many details were obtained as by-products, the rather cumbersome calculations involved are unnecessary if only the principal conclusion is desired. The general approach used here was suggested by the reviewer of the original manuscript of this paper, who attributed it to Professor C. C. Lin of the Massachusetts Institute of Technology.

\section{ON DIFFUSION IN AN EXTERNAL FIELD AND THE ADJOINT SOURCE PROBLEM*}

\section{BY JULIAN KEILSON (Lincoln Laboratory, M.I.T.)}

Abstract. If diffusion in an external field is described by $\partial \rho / \partial t=D \nabla^{2} \rho-\rho / \tau-$ $\nabla \cdot(\mathbf{F}(\mathbf{r}) \rho)$, the function $\gamma\left(r_{0}\right)$ describing the probability that a particle at $r_{0}$ will reach a collector surface before decaying or being absorbed by other surfaces satisfies the equation $D \nabla^{2} \gamma-\gamma / \tau+\mathbf{F}(\mathbf{r}) \cdot \nabla \gamma=0$. This equation has no singularity to disturb any geometric symmetry available. Boundary conditions on $\gamma(r)$ at the collector surface and other influencing surfaces are derived and shown to be independent of the external field. The boundary conditions at the secondary surfaces are homogeneous. The collector surface boundary condition is inhomogeneous.

1. Introduction. If particles with lifetime $\tau$ diffuse in the presence of an external field $\mathbf{F}(r)$, changes in density $\rho(r, t)$ are described by the continuity equation ${ }^{1}$

$$
\frac{\partial \rho}{\partial t}=-\frac{\rho}{\tau}-\nabla \cdot \mathbf{j}
$$

Here $\mathbf{j}$, the current density, is given by

$$
\mathbf{j}=-D \nabla \rho+\mathbf{F}(\mathbf{r}) \rho
$$

*Received April 17, 1953; revised manuscript received Dec. 31, 1953. The research in this document was supported jointly by the Army, Navy, and Air Force under contract with the Massachusetts Institute of Technology.

1J. Keilson, On the diffusion of decaying particles in a radial electric field, Journ. Appl. Phys., 24, 13971400 (1953). 